Philosophie ANTIQUE

\section{Philosophie antique}

Problèmes, Renaissances, Usages

19 | 2019

L'épicurisme antique

\title{
Lorenzo FERRONI, Arnaud MACÉ (éd.), Platon : Ion
}

Paris, Les Belles Lettres, 2018 (Commentario, 11), LXIV + 187 p., ISBN

978-2-25144-828-2

\section{Carlotta Capuccino}

\section{(2) OpenEdition}

\section{Journals}

Edizione digitale

URL: https://journals.openedition.org/philosant/1805

DOI: 10.4000/philosant.1805

ISSN: 2648-2789

\section{Editore}

Éditions Vrin

\section{Edizione cartacea}

Data di pubblicazione: 31 octobre 2019

Paginazione: $175-178$

ISBN: 978-2-7574-2534-3

ISSN: $1634-4561$

\section{Notizia bibliografica digitale}

Carlotta Capuccino, «Lorenzo ferronı, Arnaud macé (éd.), Platon : Ion», Philosophie antique [En ligne], 19| 2019, mis en ligne le 20 janvier 2019, consulté le 02 décembre 2022. URL : http://

journals.openedition.org/philosant/1805; DOI : https://doi.org/10.4000/philosant.1805

\section{(c) (i) $\odot$}

Creative Commons - Attribution - Pas d'Utilisation Commerciale - Pas de Modification 4.0 International - CC BY-NC-ND 4.0

https://creativecommons.org/licenses/by-nc-nd/4.0/ 
Lorenzo Ferroni, Arnaud Macé (éd.), Platon: Ion, Paris, Les Belles Lettres, 2018 (Commentario, 11), lxiv + 187 p., ISBN 978-2-25144-828-2.

A inizio estate del 2018 l'editore Les Belles Lettres di Parigi ha pubblicato una nuova «édition bilingue commentée» dello Ione platonico, la prima opera di filosofia greca antica a essere ospitata nella collana Commentario. Si tratta di un lavoro a quattro mani a cura del filologo italiano Lorenzo Ferroni e del filosofo francese Arnaud Macé, che presenta una chiara divisione dei compiti: oltre all'introduzione, alla traduzione e a un commento del dialogo (a cura di Macé), come previsto dalla sede editoriale, il volume è arricchito da un inatteso côté filologico (a cura di Ferroni) che consiste in una vera e propria edizione critica dello Ione, completa di panoramica sulla tradizione testuale del dialogo, testo greco con relativo apparato critico e note testuali di dettaglio. L'opportuna divisione delle competenze non va tuttavia a scapito dell'unità del lavoro e della condivisione delle responsabilità, unità e condivisione che gli autori dichiarano esplicitamente nell'Avertissement in apertura del volume e tengono a sottolineare attraverso l'uso esclusivo della prima persona plurale. La traduzione di Macé, in particolare, è stata rivista da entrambi gli autori e il suo contributo, in generale, è debitore alle «note» presenti nella tesi di dottorato di Ferroni, discussa a Firenze nel 2006, che già consisteva in una edizione critica dello Ione e dalla quale, infine, questo libro nel suo insieme trae origine.

L'analisi che Lorenzo Ferroni conduce in riferimento costante alla precedente edizione critica del dialogo, a cura del filologo olandese Albert Rijksbaron (Brill 2007), è dunque l'esito di un lavoro più che decennale, testimoniato dalla pubblicazione intermedia di una serie di articoli specialistici di approfondimento ai quali spesso gli autori rinviano, e rappresenta ad oggi l'unica edizione critica dello Ione che tenga conto dell'intera tradizione manoscritta medievale (in assenza di fonti papirologiche), fondandosi su nuove collazioni integrali di tutti i testimoni. Ferroni completa in questo modo il lavoro di edizione del suo predecessore Rijksbaron, che aveva condotto nuove collazioni complete dei testimoni primari, condividendone il rigore nelle analisi linguistiche, con particolare attenzione all'uso platonico delle particelle, e l'esigenza di rimeditare l'interpunzione al fine di consentire una lettura più limpida del testo. Lo stemma codicum ricostruito da Ferroni (p. 1-lvi) è di stampo classico con struttura dicotomica: dei quattro manoscritti indipendenti su cui si fonda, $\mathbf{T}$ (Venetus Marcianus Gr. App. Class. IV 1) e W (Vindobonensis Suppl. Gr. 7) si oppongono a F (Vindobonensis Suppl. Gr. 39) e, come già osservato da Rijksbaron, al manoscritto da esso indipendente S (Venetus Marcianus Gr. 189), la cui collazione integrale, sotto la supervisione esperta di Bruno Vancamp, rappresenta una delle novità di questa edizione.

Un secondo merito, oltre alla completezza dell'indagine sui manoscritti, risiede nel commento puntuale, e anche in questo caso esaustivo, delle singole varianti testuali (p. 127-163), che unisce all'expertise filologica una non comune chiarezza espositiva, rispondendo a un'esigenza editoriale: la collana Commentario si rivolge infatti a un pubblico misto di ricercatori, studenti universitari e cultori della materia a vario titolo, mettendo alla prova tanto le competenze specialistiche quanto le abilità espositive dei suoi autori. Se le note di dettaglio sono molto spesso concordi con le analisi di Rijksbaron, pur contribuendovi con l'aggiunta di nuovi argomenti a sostegno delle interpretazioni testuali condivise (si segnalano in particolare le note a 533c9 e 535b1; e per un caso di interessante disaccordo, la nota conclusiva a 542b1), è nell'orientamento interpretativo di fondo e nella sensibilità filologica che i due editori infine si allontanano. La convinzione di Rijksbaron che non sia possibile stabilire «the text which Plato 
wrote» perché non esiste alcun testo antico che sia fissato in modo definitivo, ma solo testi "fluidi" ragionevolmente ricostruibili in accordo con le regole linguistiche e culturali del periodo cui appartengono, lo porta a prediligere le lezioni più comuni e a "normalizzare" la prosa platonica sulla base di un uso del greco consolidato e regolare. L'opposto temperamento di Ferroni, che confesso a me più affine, lo guida invece nella ricerca del testo "più platonico" che siamo in grado di ricostruire, facendolo propendere, nella maggior parte dei casi, per la lectio difficilior.

Una lacuna che vorrei segnalare, in ragione di questa sensibilità filologica (ma riscontrabile già nell'edizione di Rijksbaron), riguarda gli studi di Des Places sull'uso platonico delle particelle, che a mio avviso avrebbero potuto illuminare il valore di ov̉коิvv nello Ione evitando alcune forzature nell'interpunzione del dialogo. Una scelta testuale che non condivido è invece quella della variante $\rho \alpha \psi \omega \delta$ óc $(\mathbf{T} \mathbf{W})$ in luogo di $\alpha \gamma \alpha \theta$ ò

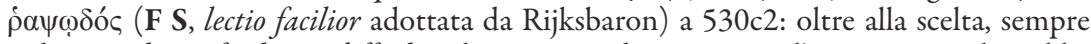
ardua, tra lectio facilior e difficilior, la necessità di mantenere l'aggettivo sembrerebbe infatti dipendere dal contesto sintattico, e in particolare dalla presenza dell'avverbio $\kappa \alpha \lambda \hat{\omega} \zeta$ al rigo $c 5$, che non è in discussione perché accolto da tutti i manoscritti. Il periodo può essere ricostruito come segue: «un buon rapsodo non potrebbe mai essere tale se non comprendesse le cose dette dal poeta! Infatti il rapsodo [scil. qualunque rapsodo] deve farsi hermeneus del pensiero del poeta per gli ascoltatori, e farlo bene senza sapere che cosa egli intenda dire è impossibile». Questo è uno di quei casi in cui, a mio parere, risulta più evidente che alle ragioni filologiche occorre affiancare un'attenta analisi logica del testo filosofico.

Arnaud Macé ha il difficile compito di introdurre gradualmente il lettore alla figura di Platone, al dialogo socratico e allo Ione, per poi procedere integrando l'analisi testuale di Ferroni con un commento continuo sezione per sezione (piuttosto che lineare, parola per parola, al modo anglosassone) delle cinque parti strutturali del dialogo e delle relative sottounità individuate su base esegetica. La tesi generale è anticipata nelle pagine introduttive, che per i due terzi ( $\mathrm{p}$. xxiii-xliii) contengono una presentazione sommaria dei temi e degli argomenti approfonditi nel commento: attraverso il dialogo tra Socrate e Ione, rapsodo omerico coronato dal successo, Platone metterebbe in scena la sua «philosophie des pratiques», il cui metodo consiste nel proporre a un «praticien» diverse rappresentazioni della sua attività, qualunque essa sia, al fine di stabilire la forma esatta di sapere che presuppone. I tre modelli sottoposti a Ione nelle tre parti del corpo dialogico, ovvero «il sapere enciclopedico» o «l'arte poetica» (530d9-533c8), «la possessione» (533c9-536d7) e «l'arte di commentare» (536d8-541d7), si rivelano egualmente fallimentari, ma ciò nonostante «le déroulement du dialogue» svolge il compito più importante di mostrare il ruolo e le modalità proprie del dialogo socratico, che nella terza parte dello Ione si presenta esso stesso intessuto di citazioni omeriche, nei «sapienti dibattiti interpretativi» che verosimilmente invitavano rapsodi, sofisti e filosofi a confrontarsi su un terreno comune. Il pregio maggiore del lavoro di Macé consiste, a mio avviso, nell'aver saputo ricostruire con ricchezza di particolari il contesto culturale specifico in cui è calata la vicenda dello Ione, grazie alle sue evidenti competenze storico-letterarie, che ben rispondono all'obiettivo della collana di «éclairer le texte» mostrandone «les enjeux esthétiques, littéraires, philosophiques et idéologique» in modo fluido, agevole e accattivante (con qualche tesi forse troppo ardita e non sufficientemente documentata, per esempio l'idea che Ione gareggiasse non solo come rapsodo ma anche servendosi di strumenti come l'aulo e la cetra, cfr. p. xxxii-xxxiii).

Mi sembra, tuttavia, che il cuore della sua proposta interpretativa, vale a dire «la filosofia delle pratiche», risenta di un fraintendimento della struttura generale del 
dialogo. Macé sembra avere chiaro, pur non citandolo in modo esplicito, il principio formulato nell' Alcibiade I (112d7-113b7) secondo cui a sostenere una tesi impegnandosi sulla sua verità è sempre l'interlocutore, ovvero chi nel dialogo ha il turno di risposta (cfr. p. 69 e n. 81). Inizialmente Socrate assume che l'attività del rapsodo consista in una techne - in una forma di sapere, dunque - perché questa è la credenza del suo interlocutore, e non perché ne sia lui stesso convinto; ma ciò non implica che tale assunzione debba essere preservata ai fini dell'argomentazione socratica. Al contrario, per Socrate si tratta di una credenza sub iudice (come Rijksbaron aveva ben colto parlando di argumenti causa, cfr. p. 10 e n. 19 della sua edizione critica), che attraverso i due esami della (presunta) techne rapsodica, prima e dopo l'analogia del magnete, sarà confutata. Non c'è allora alcun bisogno di supporre che l'ispirazione o possessione divina sia a sua volta un modello di spiegazione del sapere di Ione (il cui unico indizio sarebbe l'uso dell'aggettivo $\delta \varepsilon ı v o ́ \varsigma$, che non significa necessariamente 'abile' nel senso di 'competente', ma anche 'mirabile' o 'formidabile', con riferimento agli effetti prodotti), ma piuttosto del suo innegabile successo. La struttura dello Ione è sì tripartita, ma i modelli proposti per spiegare il successo, e non il sapere, del più celebre dei rapsodi omerici sono soltanto due: un modello gnoseologico, quello della techne, e il modello magnetico dell'ispirazione divina, ad esso alternativo. Il modello gnoseologico è presentato in due tempi attraverso l'enunciazione dei due principi necessari e sufficienti affinché una techne sia tale: il principio di unità, secondo cui la techne è un sapere unitario ( $\tau$ ò ö $\lambda \mathrm{ov}$ ) che non può essere scisso senza dissolversi (limite ante quem); e il complementare principio di individuazione, per cui ogni techne è specializzata in un solo dominio di oggetti che ne delimita il raggio d'azione (limite post quem). Confutare il primo principio, cioè una delle condizioni necessarie della techne, sarebbe bastato da un punto di vista logico, ma Ione non è persuaso e l'etica del dialogo socratico richiede che si continui a cercare l'assenso dell'interlocutore fino a che tutte le vie siano state esplorate.

Sarebbero numerosi i dettagli da discutere, a partire dalle scelte di traduzione e dalle osservazioni lessicali in genere, ma purtroppo non è possibile farlo in questa sede (per esempio trovo discutibile tradurre غ̇ंđaıvétnc con 'recitatore', perché a 536d6-7 le

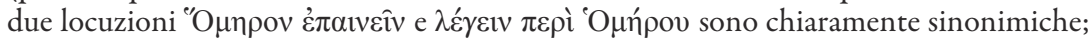

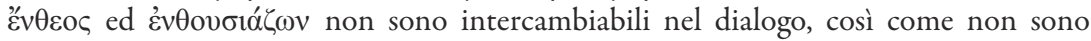

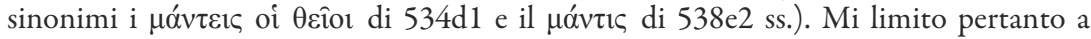
un'osservazione di carattere generale: l'impressione che ho avuto leggendo il commento di Macé è che l'autore abbia voluto proporre una propria interpretazione originale dello Ione, senza tuttavia fondarla su un confronto costante - nei modi adeguati alla sede editoriale che ospita il suo lavoro - con la letteratura critica filosofica precedente (come Ferroni ha condotto la sua edizione critica in costante riferimento ai precedenti editori), confronto dal quale certe "forzature" avrebbero potuto essere attenuate e certi debiti riconosciuti (per esempio a p. $68 \mathrm{n}$. 78 è evidente il mancato riferimento all'articolo di David L. Roochnik, «Plato's Use of ATEXN $\Omega \Sigma »$, Phoenix 41, 1987). Ciò nonostante, in virtù della sapiente ricostruzione del contesto e dei preziosi paralleli letterari, il suo commento rimane un'utile introduzione al dialogo, così come non solo è utile, ma necessario per lo specialista che voglia approfondirne l'analisi, il confronto con l'edizione critica di Ferroni, da consultare congiuntamente a quella di Rijksbaron.

Segnalo infine due refusi (p. xxii: la data di redazione dello Ione non può ovviamente essere il 490, ma semmai il 390; p. xl n. 115, passim: Jaraway sostituisce sistematicamente Janaway) e due luoghi comuni insidiosi sulla filosofia socratica (p. 70: 'sapere di non sapere niente' è formula paradossale assente nei testimoni antichi, nell'Apologia Platone scrive invece che Socrate è «consapevole di non essere sapiente»; e p. 88: l'oracolo di 
Delfi non ha proclamato Socrate «le plus savant des hommes», la sua ambiguità risiede proprio nell'insolita formulazione negativa «Nessuno è più sapiente di Socrate»).

Carlotta Capuccino

Università di Bologna

Chad Jorgenson, The Embodied Soul in Plato's Later Thought, Cambridge, Cambridge University Press, 2018 (Cambridge Classical Studies), 217 p., ISBN 978-1-107-17412-2.

En réponse à l'affirmation (ou l'accusation) d'un dualisme trop rigide tel qu'il est interprété à partir du Phédon, l'ouvrage de C. Jorgenson entend faire justice d'un ensemble de problèmes relatifs à l'âme incarnée, principalement dans la République, le Timée et le Philèbe. Peut-on envisager une analyse du composé âme-corps sur un autre mode que celui de la condamnation du corps ? L'A. fait le choix d'un parcours en « questions » en sept chapitres afin de montrer que l'intérêt de cette question de l'âme incarnée ne saurait se réduire à un examen anthropologique de moindre valeur : 1) Thymos, 2) L'âme désirante, 3) L'âme rationnelle, 4) Mesurer le plaisir, 5) Eudaimonia, 6) La sphère politique, 7) L'eschatologie. Si de fait il est difficile d'élaborer un système platonicien de l'âme incarnée, ces parcours thématiques permettent de mesurer l'écart entre un dualisme mal compris et une certaine volonté platonicienne de donner droit aux passions et opérations du vivant, qui fait état d'une autre manière, pour l'âme, d'atteindre le bien. Mélange, harmonie, équilibre, médiation sont autant de termes qui, évoquant une constitution plurielle de l'âme et de sa dimension incarnée, évoquent un dualisme d'une autre nature que le rigorisme (supposé) du Phédon.

Plutôt que de présenter l'ouvrage en fonction de ses chapitres qui sont relativement autonomes et dont la succession ne donne pas nécessairement l'image d'une thèse unifiée, on en rendra compte en fonction d'un certain nombre de thématiques transversales.

Un premier fil rouge est celui d'une supposée « évolution de la pensée de Platon ». À travers ces chapitres, l'A. répond de manière complexe à la question d'une possible évolution de la pensée platonicienne concernant le rapport entre l'âme et le corps, en isolant pour son corpus la République, le Timée et le Philèbe. Tout d'abord, contre une tradition néoplatonicienne qui fait du Phédon la pierre de touche d'un dualisme strict et définitif, au regard duquel les descriptions de l'âme incarnée dans les dialogues plus tardifs sont conçues comme des « assouplissements », l'A. montre au contraire que Platon ne cesse d'approfondir un dualisme déjà complexe qui est fait de tensions inhérentes à l'âme elle-même. Par exemple, selon l'A., l'idéal d'assimilation au divin (homoiosis theoi) qu'on pourrait voir annoncé dans le Phédon n'est pas, comme le propose D. Sedley («The ideal of godlikeness », dans G. Fine (éd.), Plato 2: Ethics, Politics, Religion, and the Soul, Oxford, Oxford University Press, 1999, p. 309-328), exclusif d'un intérêt pour les vertus démotiques et politiques pour atteindre le bonheur. De même, et en contrepoint des travaux de C. Bobonich par exemple (Plato's Utopia Recast, Oxford, Oxford University Press, 2002), l'A. montre que si évolution de Platon il y a dans la République, le Timée et le Philèbe, il ne s'agit ni de ruptures strictes (de la psychologie bipartite à la tripartition de l'âme) ni d'accommodements. En d'autres termes, le Phédon constitue pour l'A. un point de repère pour les dialogues ultérieurs concernant l'âme incarnée, mais il ne saurait constituer un point de non-retour. Une telle lecture qui est davantage unitarienne permet d'ailleurs à l'A. de convoquer utilement certains passages de dialogues « socratiques » (Protagoras, Gorgias, Banquet) qui plaident pour une plus grande continuité dans la pensée platonicienne. Il s'agit en somme de défendre 